\title{
Positron emission tomography assessment of large vessel inflammation in patients with newly diagnosed, biopsy-proven giant cell arteritis: a prospective, case-control study
}

\author{
Sergio Prieto-González, ${ }^{1}$ Marina Depetris, ${ }^{2}$ Ana García-Martínez, ${ }^{1,3}$ \\ Georgina Espígol-Frigolé, ${ }^{1}$ Itziar Tavera-Bahillo, ${ }^{1}$ Marc Corbera-Bellata, ${ }^{1}$ \\ Ester Planas-Rigol, ${ }^{1}$ Marco A Alba, ${ }^{1}$ José Hernández-Rodríguez, ${ }^{1}$ Josep M Grau, ${ }^{4}$ \\ Franciso Lomeña, ${ }^{2}$ Maria C Cid ${ }^{1}$
}

Handling editor Tore K Kvien

- Additional material is published online only. To view please visit the journal online (http://dx.doi.org/10.1136/ annrheumdis-2013-204572).

For numbered affiliations see end of article.

\section{Correspondence to}

Dr Maria C Cid

Department of Autoimmune

Diseases, Clinical Institute of

Medicine and Dermatology,

Hospital Clínic, Villarroel 170,

Barcelona 08036, Spain;

mccid@clinic.ub.es

$\mathrm{FL}$ and MCC share senior authorship.

Results presented at the European Congress of Rheumatology (EULAR) 2013, Madrid, Spain, June 2013, and at the Annual Congress of European Association of Nuclear Medicine, Lyon, France, October 2013.

Received 7 September 2013 Revised 13 February 2014 Accepted 1 March 2014 Published Online First 24 March 2014

\section{(a) CrossMark}

To cite: Prieto-González S, Depetris M, García-

Martínez $\mathrm{A}$, et al. Ann

Rheum Dis 2014:73:1388-

1392.

\section{ABSTRACT}

Background Positron emission tomography (PET) scan is emerging as a promising imaging technique to detect large-vessel inflammation in giant cell arteritis (GCA). However, the lack of a standardised definition of arteritis based on ${ }^{18}$ fluorodeoxyglucose (FDG) uptake is an important limitation to the use of PET scan for diagnostic purposes.

Objective To prospectively assess the intensity and distribution of FDG uptake at different vascular territories in patients with newly diagnosed GCA compared with controls.

Methods 32 consecutive, biopsy-proven, GCA patients treated with glucocorticoids for $\leq 3$ days were included. The control group consisted of 20 individuals, who underwent PET/CT for cancer staging. Maximal standardised uptake value (SUVm) was calculated at four aortic segments, supraaortic branches and iliac-femoral territory. Sensitivity and specificity was calculated by receiver-operator characteristic curves (ROC) analysis. Results Mean SUVm was significantly higher in patients than in controls in all vessels explored and correlated with acute-phase reactants and serum IL-6. Mean of the SUVm at all the vascular territories had an area under the curve (AUC) of 0.830 , and a cut-off of 1.89 yielded a sensitivity of $80 \%$ and a specificity of $79 \%$ for GCA diagnosis.

There were no significant differences in AUC among the vascular beds examined.

Conclusions FDG uptake by large vessels has a substantial sensitivity and specificity for GCA diagnosis.

\section{INTRODUCTION}

Temporal artery biopsy is the gold standard for the diagnosis of giant cell arteritis (GCA) due to the tropism of GCA for the epicranial arteries. ${ }^{1}{ }^{2}$ With a few exceptions, ${ }^{3}$ histopathological demonstration of temporal artery inflammation provides the most definitive evidence of GCA. Doppler ultrasonography (DUS) of temporal arteries has emerged as a useful alternative tool in centres where biopsy is not easily available. ${ }^{1}$

The diagnosis of GCA may be also supported by demonstrating extracranial artery involvement by imaging. Over the past recent years, positron emission tomography/CT (PET/CT), CT angiography, magnetic resonance imaging (MRI) angiography and DUS have revealed that extracranial involvement in GCA is more frequent than previously anticipated, occurring in $30-74 \%$ of patients. ${ }^{4-7}$

PET detection of large-vessel involvement in patients with fever of unknown origin, unexplained constitutional symptoms or apparently isolated polymyalgia rheumatica (PMR) has emphasised its diagnostic potential. ${ }^{8} 9$ A limitation of PET as a diagnostic tool is the lack of a standardised definition of vascular inflammation based on the intensity of ${ }^{18}$ fluorodeoxyglucose (FDG) uptake. While visual assessment of intensively positive cases may be clear, there is no consensus about the minimal intensity of FDG uptake necessary to define vascular inflammation. Conversely, atherosclerosis and ageing may increase vascular FDG uptake, potentially leading to vasculitis overdiagnosis. ${ }^{10}$

In this study, we measured FDG uptake by different vascular territories in a cohort of newly diagnosed patients and controls and performed receiver-operator characteristic curves (ROC) analysis to determine sensitivity and specificity of FDG uptake to detect inflammation at different vascular sites. As a secondary endpoint, we analysed potential correlation between FDG uptake and inflammatory biomarkers.

\section{MATERIALS AND METHODS}

\section{Patients}

Between November 2006 and March 2011, all patients diagnosed with biopsy-proven $\mathrm{GCA}^{2}$ at our institution were assessed for potential participation in the study. Patients who had received glucocorticoid treatment for $>3$ days were excluded. Clinical and laboratory data recorded are detailed in the online supplementary methods. The study was approved by the ethics committee (Hospital Clínic, Barcelona).

The control group included 20 patients with no chronic inflammatory diseases, matched for gender, age and cardiovascular risk factors (CVRF), consecutively selected among patients who underwent PET/CT during the same timeframe for early lung cancer staging. 


\section{PET/CT protocol}

PET scans were performed using a hybrid PET/CT (Biograph, Siemens) with an ECAT EXACT HR+BGO PET and a helicoidal CT scanner (Somatom, Emotion). Patients fasted $4 \mathrm{~h}$ before injection of $370 \mathrm{MBq}$ of 18F-FDG. Whole-body PET data were acquired $60 \mathrm{~min}$ after in three-dimensional mode and for $5 \mathrm{~min}$ per bed position. PET images were reconstructed both with and without CT data for attenuation correction. A region of interest (ROI) in 3-D around the vessel was placed manually in transaxial, sagittal and coronal slices. The standardised uptake value (SUV) was calculated based on the measured activity, decay-corrected injected dose and patient body weight. $\mathrm{SUVm}=$ maximal activity (ROI) $(\mathrm{mBq} / \mathrm{mL}) /$ injected dose $(\mathrm{mBq}) /$ weight $(\mathrm{g})$.

Four aortic segments (ascending thoracic aorta, aortic arch, descending thoracic aorta and abdominal aorta) and the main tributaries-carotid, subclavian, axillary, iliac and femoral arteries (each bilaterally) - were evaluated. The control group was subjected to the same PET/CT protocol. Assessment of PET data was carried out by two nuclear medicine specialists (FL and $\mathrm{MD}$ ), who were blinded to clinical and pathological findings. However, unequivocal masked evaluation could not be guaranteed due to the controls' disease.

\section{Statistical analysis}

ROC were applied to each vascular territory to calculate sensitivity and specificity. Area under the curve (AUC) comparison was performed by Hanley and McNeil analysis. Cut-offs with best sensitivity and specificity were selected. Mann-Whitney $U$ test or Student $t$ test, when applicable, were used for quantitative data. Correlations were calculated using Pearson's or Spearman's test. Statistical significance was defined as $p<0.05$. Calculations were performed with the IBM SPSS Statistics (V.20.0, Armonk, New York, USA).

\section{RESULTS}

\section{Clinical and laboratory findings of the GCA cohort}

Seventy-one GCA patients were diagnosed during the recruitment period. Eight patients refused participation, and 31 had received glucocorticoid treatment for $>3$ days. The remaining 32 were included. Seventeen of them had been treated for $\leq 3$ days at the time of imaging. Treatment consisted of oral prednisone at $1 \mathrm{mg} / \mathrm{kg} /$ day. Two patients received $250 \mathrm{mg}$ intravenous methylprednisolone pulses ( 1 and 7 pulses, respectively) due to severe cranial ischaemic symptoms.

Online supplementary table S1 shows the clinical and laboratory data of the study group. There were no relevant differences in age, gender or CVRF between patients and controls (see online supplementary table S2).

\section{FDG uptake cut-off for GCA diagnosis}

SUVm at any vascular territory explored was significantly higher in GCA patients than in controls (table 1). ROC curves and AUCs are displayed in figure 1 and table 1 , respectively. Mean of the SUVm observed at all the vascular territories had an AUC of $0.830(0.715-0.946)$. A cut-off of 1.89 had a sensitivity of $80 \%$ and a specificity of $79 \%$. Mean of the SUVm at supraaortic vessels showed the highest AUC (0.832). In this site, a cut-off of 1.70 achieved a sensitivity and specificity of 81 and $79 \%$, respectively, for the diagnosis of GCA (95\% CI 0.720 to 0.946 ). FDG uptake at the aorta showed lower AUC (0.738), with a sensitivity and specificity of 90 and 42, respectively, using a cut-off of 2.25 , and a sensitivity of $58 \%$, specificity of $90 \%$ with a cut-off of 2.65 (95\% CI 0.598 to 0.881$)$. However, differences in AUCs among territories did not reach statistical significance.

Vascular/liver uptake ratios were also significantly higher in patients than in controls at the right axillary and carotid arteries,

Table 1 SUVm and AUC at each vascular bed assessed

\begin{tabular}{|c|c|c|c|c|}
\hline Territory & GCA patients (mean \pm SD) & Controls $(m e a n \pm S D)$ & $\mathrm{p}$ Value & AUC $(95 \% \mathrm{Cl})$ \\
\hline Ascending aorta & $2.63 \pm 0.57$ & $2.17 \pm 0.26$ & $<0.001$ & 0.778 (0.651 to 0.904$)$ \\
\hline Aortic arch & $2.61 \pm 0.50$ & $2.23 \pm 0.31$ & 0.002 & $0.756(0.621$ to 0.891$)$ \\
\hline Descending thoracic aorta & $2.78 \pm 0.65$ & $2.39 \pm 0.33$ & 0.007 & 0.739 (0.598 to 0.881$)$ \\
\hline Abdominal aorta & $2.97 \pm 0.60$ & $2.56 \pm 0.39$ & 0.005 & $0.748(0.608$ to 0.888$)$ \\
\hline Right subclavian artery & $2.46 \pm 0.54$ & $2.14 \pm 0.40$ & 0.030 & 0.763 (0.607 to 0.889$)$ \\
\hline Left subclavian artery & $2.26 \pm 0.56$ & $1.89 \pm 0.28$ & 0.003 & $0.764(0.610$ to 0.891$)$ \\
\hline Right carotid artery & $2.33 \pm 0.52$ & $1.83 \pm 0.25$ & $<0.001$ & $0.812(0.695$ to 0.930$)$ \\
\hline Left carotid artery & $2.32 \pm 0.51$ & $1.97 \pm 0.30$ & 0.004 & 0.733 (0.594 to 0.872 ) \\
\hline Right axillary artery & $1.21 \pm 0.31$ & $0.88 \pm 0.17$ & $<0.001$ & $0.830(0.725$ to 0.940$)$ \\
\hline Left axillary artery & $1.09 \pm 0.34$ & $0.88 \pm 0.18$ & 0.001 & 0.780 (0.627 to 0.886$)$ \\
\hline Right iliac artery & $2.41 \pm 0.67$ & $2.01 \pm 0.38$ & 0.009 & 0.747 (0.606 to 0.888$)$ \\
\hline Left iliac artery & $2.46 \pm 0.47$ & $2.00 \pm 0.41$ & 0.002 & $0.767(0.628$ to 0.905$)$ \\
\hline Right femoral artery & $1.68 \pm 0.39$ & $1.24 \pm 0.22$ & $<0.001$ & $0.817(0.715$ to 0.928$)$ \\
\hline Left femoral artery & $1.50 \pm 0.37$ & $1.14 \pm 0.18$ & $<0.001$ & 0.801 (0.679 to 0.922$)$ \\
\hline All territories* & $2.15 \pm 0.37$ & $1.79 \pm 0.17$ & $<0.001$ & $0.830(0.715$ to 0.946$)$ \\
\hline Aorta** & $2.75 \pm 0.54$ & $2.34 \pm 0.23$ & 0.001 & $0.738(0.612$ to 0.874$)$ \\
\hline Supraaortic branches** & $1.95 \pm 0.35$ & $1.59 \pm 0.15$ & $<0.001$ & 0.832 ( 0.732 to 0.968$)$ \\
\hline Iliofemoral territory** & $1.97 \pm 0.36$ & $1.62 \pm 0.23$ & $<0.001$ & $0.802(0.679$ to 0.925$)$ \\
\hline Liver & $2.76 \pm 0.57$ & $2.52 \pm 0.42$ & 0.119 & $0.635(0.480$ to 0.790$)$ \\
\hline
\end{tabular}



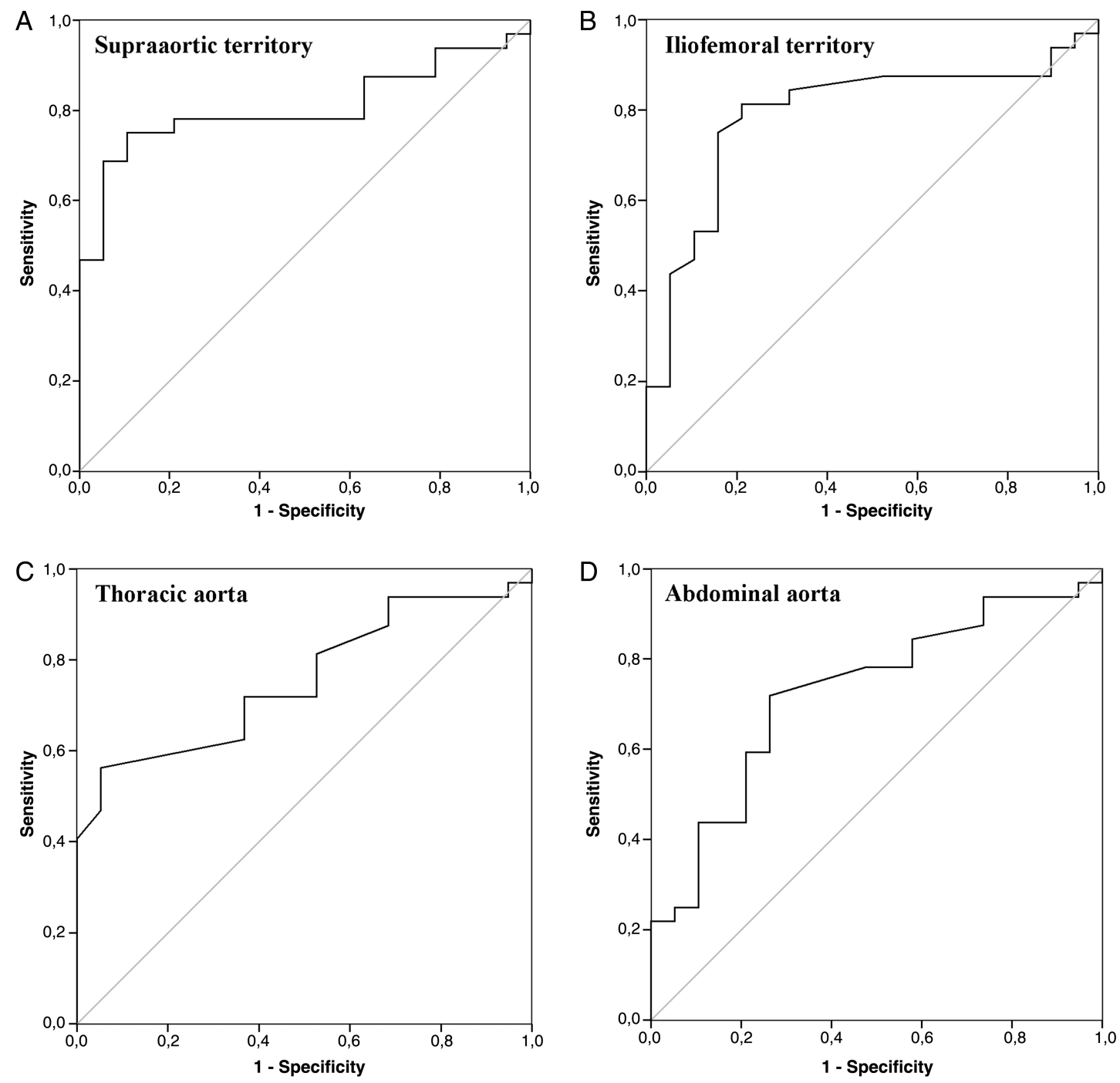

Figure 1 Receiver-operator characteristic curves of standardised uptake value at different vascular regions.

but the overall discriminatory performance was much lower (see online supplementary table S3).

\section{Relationship between FDG uptake and clinical and laboratory findings}

Patients with cranial symptoms presented significantly higher values of maximal and mean SUVm (combined average of all vascular territories) than patients lacking cranial manifestations. No relationship between the intensity of FDG uptake and other clinical findings was observed (table 2). No differences in maximal or mean SUVm were observed between treatment-naïve patients and those who had received glucocorticoids. The maximal and mean SUVm correlated with acute-phase reactants and serum IL-6 concentrations (table 2).

\section{DISCUSSION}

The present prospective study, performed in an unselected patient cohort with unequivocal GCA, shows that FDG uptake is significantly stronger in patients than in controls in all vascular territories tested, confirming the diagnostic potential of PET/ $\mathrm{CT}^{4} 8911 \mathrm{PET} / \mathrm{CT}$ allows rapid, reproducible and broad vascular evaluation. Nevertheless, there is no standardised definition of vasculitis based on an objective FDG uptake measure, and strategies employed to establish a PET-based diagnosis of GCA has been heterogeneous. Most studies have used qualitative visual assessment or a semiquantitative score using liver uptake as a reference. Visual scoring has a remarkable investigator dependency and interobserver variability. Liver uptake is influenced by individual metabolic activity, glucocorticoid treatment and the time lapse between injection and scanning. ${ }^{12}$

We tried to overcome this limitation by objectively quantifying FDG uptake by different vascular beds in patients and controls and performing ROC analysis to determine the optimal cut-off for GCA diagnosis at different vascular territories. FDG uptake by supraaortic branches had the highest AUC, in accordance with a pioneer study showing that supraaortic branches were the most frequently involved when assessed by PET. ${ }^{4}$ In this area, an FDG uptake cut-off value of 1.70 had the best sensitivity and specificity. A similar value, in the same territory but with lower performance $(\mathrm{AUC}=0.72$ ), was reported in a retrospective study of 17 patients with GCA and 3 Takayasu arteritis patients. ${ }^{13}$ This observation may be useful to differentiate GCA from other inflammatory aortic diseases that may produce systemic complaints and active aortic FDG uptake, including idiopathic aortitis, periaortitis, IgG4 disease and severe atherosclerosis. ${ }^{10}{ }^{14-17}$ This is crucial since a positive PET/CT may be accepted in the near future as a diagnostic criterion and is currently accepted as such in an ongoing clinical trial with tocilizumab in GCA. ${ }^{18}$ 
Table 2 Relationship between clinical and laboratory data and maximal SUV at any vascular territory (SUVm) and mean of the SUVm obtained at every vascular bed assessed (mean SUVm).

\begin{tabular}{|c|c|c|c|c|}
\hline & \multicolumn{2}{|l|}{ Maximal SUVm } & \multicolumn{2}{|l|}{ Mean SUVm } \\
\hline & & p Value & & p Value \\
\hline Cranial symptoms (P/A) & $3.21 \pm 0.65 / 2.50 \pm 0.52$ & 0.021 & $2.24 \pm 0.32 / 1.77 \pm 0.36$ & 0.004 \\
\hline Systemic symptoms (P/A) & $3.12 \pm 0.61 / 2.98 \pm 0.82$ & 0.589 & $2.20 \pm 0.35 / 2.07 \pm 0.43$ & 0.354 \\
\hline Ischaemic symptoms (P/A) & $2.91 \pm 0.61 / 3.14 \pm 0.71$ & 0.402 & $2.11 \pm 0.39 / 2.17 \pm 0.37$ & 0.708 \\
\hline PMR (P/A) & $3.11 \pm 0.62 / 3.06 \pm 0.71$ & 0.886 & $2.27 \pm 0.35 / 2.12 \pm 0.38$ & 0.321 \\
\hline $\mathrm{GC}$ treatment $(\mathrm{Y} / \mathrm{N})$ & $2.97 \pm 0.44 / 3.20 \pm 0.81$ & 0.385 & $2.14 \pm 0.36 / 2.17 \pm 0.40$ & 0.858 \\
\hline CRP, mg/dL & $r=0.551$ & 0.001 & $r=0.476$ & 0.034 \\
\hline $\mathrm{ESR}, \mathrm{mm} / \mathrm{h}$ & $r=0.442$ & 0.011 & $r=0.335$ & 0.050 \\
\hline Haptoglobin, mg/dL & $r=0.585$ & 0.008 & $r=0.358$ & 0.050 \\
\hline IL-6, pg/mL & $r=0.616$ & 0.002 & $r=0.544$ & 0.007 \\
\hline
\end{tabular}

The sensitivity and specificity of PET/CT obtained in this study is close to that calculated in a recent systematic review/ meta-analysis of heterogeneous, mostly retrospective studies, and those reported in a retrospective analysis evaluating the impact of PET on the management of patients with suspected large-vessel vasculitis. ${ }^{19}$

Interestingly, FDG uptake by the aorta showed a lower AUC, being worse in the abdominal segment where atherosclerosis is more prevalent in the general population. This fact highlights the diagnostic limitation of PET in this territory since aortic FDG uptake may be markedly influenced by ageing or atheroma plaques. Hautzel et al reported a higher sensitivity and specificity of thoracic aorta FDG uptake to detect large-vessel inflammation in a cohort of 18 patients with GCA. ${ }^{20} \mathrm{~A}$ thoracic aorta/ liver ratio of 1.0 had a sensitivity and specificity of $88 \%$ and $93 \%$, respectively (AUC $=0.932$ ). However, a substantial proportion of the patients assembled in this cohort were selected on the basis of previously known large-vessel involvement demonstrated by other techniques. In our study, direct, territoryfocused comparison of SUVm between patients and controls discriminated better than vascular/liver ratios.

A retrospective study evaluating how PET/CT results influenced management of patients with suspected GCA suggested that previous glucocorticoid (GC) treatment decreased the diagnostic yield of PET/CT. ${ }^{19}$ Sequential assessments have demonstrated, indeed, that FDG uptake decreases after 3 months of treatment. ${ }^{4}$ The present study suggests that a short course of therapy ( $\leq 3$ days) may not substantially reduce the diagnostic accuracy of PET/CT.

In conclusion, this study provides sensitive and specific, territory-focused cut-off values to detect vascular inflammation by PET/CT. A limitation of the study is that while patients were prospectively recruited, controls were retrospectively selected. Another limitation is the relatively small number of patients analysed, although our cohort is among the largest investigated. Further prospective studies using objective cut-offs are necessary to confirm their diagnostic performance in patients with suspected GCA.

\section{Author affiliations}

${ }^{1}$ Vasculitis Research Unit, Department of Systemic Autoimmune Diseases, Hospital Clínic, University of Barcelona, Institut d'Investigacions Biomèdiques August Pi i Sunyer (IDIBAPS), Barcelona, Spain

${ }^{2}$ Center for Diagnostic Imaging, Hospital Clínic, University of Barcelona, Institut d'Investigacions Biomèdiques August Pi i Sunyer (IDIBAPS), Barcelona, Spain
${ }^{3}$ Department of Emergency Medicine, Hospital Clínic, University of Barcelona, Institut $d^{\prime}$ Investigacions Biomèdiques August Pi i Sunyer (IDIBAPS), Barcelona, Spain ${ }^{4}$ Department of Internal Medicine, Hospital Clínic, University of Barcelona, Institut $\mathrm{d}^{\prime}$ Investigacions Biomèdiques August Pi i Sunyer (IDIBAPS), Barcelona, Spain

Contributors MCC had full access to all of the data in the study and takes responsibility for the integrity of the data and the accuracy of the data analysis. Study design: SP-G, FL and MCC. Acquisition of data: SP-G, MD, AG-M, GE-F, IT-B, $F L, M C-B, E P-R, M A A, J M G, J H-R$ and MCC. Analysis and interpretation of data: SP-G, MD, GE-F, FL, JH-R and MCC. Manuscript preparation: SP-G, FL, JH-R, JMG and MCC. Statistical analysis: SP-G, MAA, MCC.

Competing interests Supported by Ministerio de Economía y Competitividad (SAF 08/04328 and SAF11/30073). SP-G was a postresidency research award recipient from Hospital Clínic. MAA was supported by Consejo Nacional de Ciencia y Tenología (CONACyT), Mexico, and by Agencia de Gestió d'Ajuts Universitaris i de Recerca (AGAUR), Generalitat de Catalunya.

Ethics approval The study was approved by the ethics committee of our institution (Hospital Clínic, Barcelona).

Provenance and peer review Not commissioned; externally peer reviewed.

\section{REFERENCES}

1 Salvarani C, Pipitone N, Versari A, et al. Clinical features of polymyalgia rheumatica and giant cell arteritis. Nat Rev Rheumatol 2012;8:509-21.

2 Lie JT. Illustrated histopathologic classification criteria for selected vasculitis syndromes. American College of Rheumatology Subcommittee on Classification of Vasculitis. Arthritis Rheum 1990;33:1074-87.

3 Esteban MJ, Font C, Hernandez-Rodriguez J, et al. Small-vessel vasculitis surrounding a spared temporal artery: clinical and pathological findings in a series of twenty-eight patients. Arthritis Rheum 2001;44:1387-95.

4 Blockmans D, de Ceuninck L, Vanderschueren S, et al. Repetitive $18 \mathrm{~F}$-fluorodeoxyglucose positron emission tomography in giant cell arteritis: a prospective study of 35 patients. Arthritis Rheum 2006;55:131-7.

5 Prieto-Gonzalez S, Arguis P, Garcia-Martinez A, et al. Large vessel involvement in biopsy-proven giant cell arteritis: prospective study in 40 newly diagnosed patients using CT angiography. Ann Rheum Dis 2012;71:1170-6.

6 Schmidt WA, Seifert A, Gromnica-Ihle E, et al. Ultrasound of proximal upper extremity arteries to increase the diagnostic yield in large-vessel giant cell arteritis. Rheumatology (Oxford) 2008;47:96-101.

7 Aschwanden M, Kesten F, Stern M, et al. Vascular involvement in patients with giant cell arteritis determined by duplex sonography of $2 \times 11$ arterial regions. Ann Rheum Dis 2010;69:1356-9.

8 Hao R, Yuan L, Kan Y, et al. Diagnostic performance of 18F-FDG PET/CT in patients with fever of unknown origin: a meta-analysis. Nucl Med Commun 2013; 34:682-8.

9 Blockmans D, De Ceuninck L, Vanderschueren S, et al. Repetitive 18-fluorodeoxyglucose positron emission tomography in isolated polymyalgia rheumatica: a prospective study in 35 patients. Rheumatology (Oxford) 2007;46:672-7.

10 Dunphy MP, Freiman A, Larson SM, et al. Association of vascular 18F-FDG uptake with vascular calcification. J Nucl Med 2005;46:1278-84. 


\section{Clinical and epidemiological research}

11 Besson FL, Parienti JJ, Bienvenu B, et al. Diagnostic performance of (1) F-fluorodeoxyglucose positron emission tomography in giant cell arteritis: a systematic review and meta-analysis. Eur J Nucl Med Mol Imaging 2011;38:1764-72.

12 Iozzo P, Geisler $F$, Oikonen $V$, et al. Insulin stimulates liver glucose uptake in humans; an 18F-FDG uptake in humans. J Nucl Med 2003;

44:682-9.

13 Lehmann P, Buchtala S, Achajew N, et al. 18F-FDG PET as a diagnostic procedure in large vessel vasculitis-a controlled, blinded re-examination of routine PET scans. Clin Rheumatol 2011;30:37-42.

14 Reeps C, Essler M, Pelisek J, et al. Increased 18F-fluorodeoxyglucose uptake in abdominal aortic aneurysms in positron emission/computed tomography is associated with inflammation, aortic wall instability, and acute symptoms. J Vasc Surg 2008;48:417-23.
15 Salvarani C, Pipitone N, Versari A, et al. Positron emission tomography (PET): evaluation of chronic periaortitis. Arthritis Rheum 2005;53:298-303.

16 Vaglio A, Catanoso MG, Spaggiari L, et al. IL6 as an inflammatory mediator and target of therapy in chronic periaortitis. Arthritis Rheum 2013;65:2469-75.

17 Vaglio A, Salvarani C, Buzio C. Retroperitoneal fibrosis. Lancet 2006; 367:241-51.

18 Unizony SH, Dasgupta B, Fisheleva $\mathrm{E}$, et al. Design of the tocilizumab in giant cell arteritis trial. Int J Rheumatol 2013;2013:912562.

19 Fuchs M, Briel M, Daikeler T, et al. The impact of 18F-FDG PET on the management of patients with suspected large vessel vasculitis. Eur I Nucl Med Mol Imaging 2012;39:344-53.

20 Hautzel $\mathrm{H}$, Sander $\mathrm{O}$, Heinzel $\mathrm{A}$, et al. Assessment of large-vessel involvement in giant cell arteritis with 18F-FDG PET: introducing an ROC-analysis-based cutoff ratio. J Nucl Med 2008:49:1107-13. 


\section{ARD Positron emission tomography assessment} of large vessel inflammation in patients with newly diagnosed, biopsy-proven giant cell arteritis: a prospective, case-control study

Sergio Prieto-González, Marina Depetris, Ana García-Martínez, Georgina Espígol-Frigolé, Itziar Tavera-Bahillo, Marc Corbera-Bellata, Ester Planas-Rigol, Marco A Alba, José Hernández-Rodríguez, Josep M Grau, Franciso Lomeña and Maria C Cid

Ann Rheum Dis 2014 73: 1388-1392 originally published online March 24, 2014

doi: 10.1136/annrheumdis-2013-204572

Updated information and services can be found at:

http://ard.bmj.com/content/73/7/1388

Supplementary Material

References

Email alerting service
These include:

Supplementary material can be found at:

http://ard.bmj.com/content/suppl/2014/03/24/annrheumdis-2013-2045 72.DC1

This article cites 19 articles, 7 of which you can access for free at: http://ard.bmj.com/content/73/7/1388\#BIBL

Receive free email alerts when new articles cite this article. Sign up in the box at the top right corner of the online article.

\section{Collections}

Articles on similar topics can be found in the following collections

Clinical diagnostic tests (1282)

Immunology (including allergy) (5144)

Radiology (1113)

Radiology (diagnostics) (750)

Vascularitis (294)

Inflammation (1251)

Epidemiology (1367)

\section{Notes}

To request permissions go to:

http://group.bmj.com/group/rights-licensing/permissions

To order reprints go to:

http://journals.bmj.com/cgi/reprintform

To subscribe to BMJ go to:

http://group.bmj.com/subscribe/ 